EGU21-15202

https://doi.org/10.5194/egusphere-egu21-15202

EGU General Assembly 2021

(c) Author(s) 2021. This work is distributed under

the Creative Commons Attribution 4.0 License.

\title{
Earthquake rupture properties in presence of thermal -pressurization of pore fluids
}

\author{
P. Martin Mai ${ }^{1}$, Jagdish Vyas ${ }^{1}$, Alice-Agnes Gabriel ${ }^{2}$, and Thomas Ulrich ${ }^{2}$ \\ ${ }^{1}$ King Abdullah University of Science \& Technology, Earth Sciences and Engineering, Physical Science \& Engineering, Thuwal, \\ Saudi Arabia (martin.mai@kaust.edu.sa) \\ ${ }^{2}$ Ludwig Maximilian University, Munich
}

Frictional heat generated in the fault core during earthquake rupture can raise the fluid pressure in the slip zone. Such increase of fluid pressure decreases the effective normal stress and thereby lowers the frictional strength of the fault. Therefore, thermal pressurization (TP) of pore fluid affects earthquake rupture processes including nucleation, propagation, and arrest. While the effects of pore pressure and fluid flow rate on dynamic weakening of faults are qualitatively understood, a detailed analysis of how TP affects earthquake rupture parameters is needed to further deepen our understanding.

In this study, we investigate the role of two key TP parameters -- hydraulic diffusivity and shearzone half-width -- earthquake dynamics and kinematic source properties (slip, peak slip-rate, rupture speed and rise time). We conduct a suite of 3D dynamic rupture simulations applying a rate-and-state dependent friction law (with strong velocity weakening) coupled with thermalpressurization of pore fluids. Simulations are carried out with the open source software SeisSol (www.seissol.org). The temporal evolution of rupture parameters over $\sim 1$ '000 randomly distributed on-fault receivers is statistically analyzed in terms of mean variations of rupture parameters and correlations among rupture parameters.

Our simulations reveal that mean slip decreases with increasing hydraulic diffusivity, whereas mean peak slip-rate and rupture speed remain nearly constant. On the other hand, we observe only a slight decrease of mean slip with increasing shear-zone half-width, whereas mean peak sliprate and rupture speed show clear decrease. The faster diffusion of pore pressure as hydraulic diffusivity increases promotes faster increase of the effective normal stress (and fault strength) behind the main rupture front, reducing the rise time and, therefore also affecting mean slip. An increase in shear-zone half- width represents a heat source distributed over larger fault normal distance causing a second-order effect on mean slip. Additionally, our simulations reveal correlations among rupture parameters: 1) slip has weak negative correlation with peak slip-rate and negligible correlation with rupture speed, but a positive correlation with rise time, 2) peak sliprate has a strong positive correlation with rupture speed, but a strong negative correlation with rise time, 3) rupture speed has strong negative correlation with rise time. We observe little or negligible effects of variations of hydraulic diffusivity and shear-zone half- width on the 
correlations between rupture parameters. Overall, our study builds a fundamental understanding on how thermal pressurization of pore fluids affects dynamic and thereby kinematic earthquake rupture properties. Our findings are thus important for the earthquake source modeling community, and particularly, for assessing seismic hazard due to induced events in geo-reservoirs. 